\title{
Anesthesia Management in Case of Carotid Anatomic Variant during Carotid Endarterectomy under General Anesthesia
}

\author{
David Faraoni ${ }^{1}$, Benjamin Mine ${ }^{2}$, Giulia Apicella ${ }^{3}$, Alexandre Joosten ${ }^{4}$ \\ ${ }^{1}$ Department of Anesthesiology, Centre Hospitalier Universitaire Brugmann, Université Libre de Bruxelles, Brussels, Belgium; \\ ${ }^{2}$ Department of Diagnostic \& Interventional Neuroradiology, Erasme University Hospital, Université Libre de Bruxelles, Brussels, \\ Belgium; ${ }^{3}$ Department of Vascular Surgery, Erasme University Hospital, Brussels, Belgium; ${ }^{4}$ Department of Anesthesiology, Erasme \\ University Hospital, Université Libre de Bruxelles, Brussels, Belgium. \\ Email: David.FARAONI@huderf.be
}

Received February $4^{\text {th }}, 2012$; revised February $26^{\text {th }}, 2012$; accepted March $4^{\text {th }}, 2012$

\begin{abstract}
Carotid endarterectomy is an established treatment in the prevention of transient ischemic attack or stroke. Cerebral embolism, thrombosis or hypo-perfusion will lead to a major risk of perioperative thrombotic events. In this case, we report an interesting anesthesia approach for aortic arch anatomic variant management and monitoring.
\end{abstract}

Keywords: Neuromonitoring; Vascular Surgery; Carotid Surgery; Stroke; Bispectral Index

\section{Introduction}

Carotid endarterectomy (CEA) is an established treatment in the prevention of transient ischemic attack (TIA) or stroke, when symptomatic or severe asymptomatic carotid artery stenosis is diagnosed [1]. Cerebral embolism, thrombosis or hypo-perfusion will lead to a major risk of perioperative TIA with a reported incidence varying between $2 \%$ and $10 \%$ [2]. Anesthesia technique affects outcome and is currently under evaluation in a largescale study of general anesthesia versus local anesthesia. In this report, an original case associating aortic arch anatomic variant and TIA after selective carotid shunt insertion is described. It opens the discussion about anesthesia management in case of aortic arch anatomic variant.

\section{Case Description}

A 53 years old female was admitted in our vascular surgery department and scheduled for a left CEA. The patient presented a recent history of TIA leading to a left carotid stenosis diagnosis. Preoperative arterial Doppler scan revealed a significant occlusion $(>70 \%)$ of the left main carotid and was confirmed by Magnetic Resonance Angiography (MRA). On the opposite site, atheromatosis was described but no significant stenosis was diagnosed. As usual in our cardiovascular anesthesia clinics, surgery was performed under general anesthesia. After induction with remifentanil Target controlled infusion (TCI) and a bolus of etomidate, sevoflurane was chosen as maintenance agent and titrated according to Bispectral index (BIS) values. Remifentanil TCI was titrated according to hemodynamic parameters. Monitoring consisted of a five-leads electrocardiogram, pulse oxymetry, non-invasive blood pressure and invasive blood pressure measurement with a right radial artery catheter. Bispectral index probe (BIS Quatro TM, Aspect Medical Systems, Newton, MA, USA and Leiden, The Netherlands) was voluntarily added on the opposite side of the forehead corresponding to the occluded carotid with an aim to monitor hypnosis and avoid possible ischemia artifact. BIS (BIS X for Dräger, Aspect Medical System. Inc, Norwood, US) and other parameters (Dräger Infinity M540, Dräger Medical GmbH, Lübeck, Germany) were recorded with a Zeus ${ }^{\circledR}$ Infinity Empowered Anesthesia Machine (Dräger, Dräger Medical GmbH, Lübeck, Germany). BIS, Signal Quality Index (SQI), suppression ratio (SR) and unprocessed EEG waveform were continuously analyzed. Moreover, a multichannel electroencephalogram (9 electrodes placed according on N, Fp1, Fp2, A1, A2, G1, G2, C3, C4) was added after induction of anesthesia and interpreted by an experiment neurologist before and during the whole duration of carotid cross clamping (CXC). After incision and cervical dissection, the left carotid was exposed and clamped under continuous multichannel EEG monitoring. Some seconds later, 
the neurologist has detected EEG ischemic pattern in the left hemisphere. Then, CXC was removed and the decision to insert an intraluminal shunt was taken. During the second attempt of CXC, the right radial artery waveform disappeared and appeared occlusive when the shunt was inserted. Moreover, an ischemic EEG pattern was detected on the right hemisphere and the BIS value measured on the right frontal area dropped from 45 to 35 . According to this particular association, the proximal part of the shunt was removed back and the right radial arterial waveform reappeared. Bilateral EEG monitoring returned to a normal pattern associated with general anesthesia. Figure 1 shows the unprocessed EEG before and after shunt mobilization. Figure 2 shows the MRA image which was immediately checked in the OR showing that the brachiocephalic trunk and the left carotid artery present a common insertion on the aortic arch, so-called Bovine Aortic Arch Variant [3]. This allows us to understand that the shunt was clearly inserted too deeply in the proximal part of the left carotid leading to the occlusion of the brachiocephalic trunk. At the end of surgery, the patient recovery was clear without any neurological deficit. The patient was transferred to the postanesthesia care unit and monitored during 24 hours. No postoperative neurological dysfunction was evidenced.

\section{Discussion}

Different great vessels anatomic variants are described in the literature. In our case, innominate trunk was born from the left common carotid artery. This presentation, called "Bovine Aortic Arch Variant", is usual (incidence rate: $20 \%$ ) and appeared to be without clinical importance [4]. In our case, the misdiagnosis of this particular presentation could have led to an ischemic complication in the perioperative period. For this reason, management of anatomic variant appears crucial in the preoperative

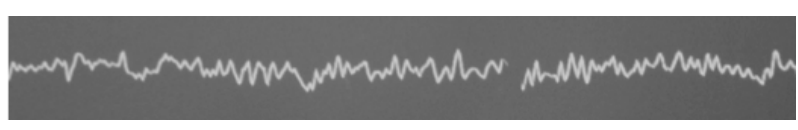

(a)

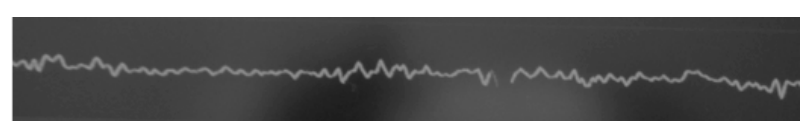

(b)

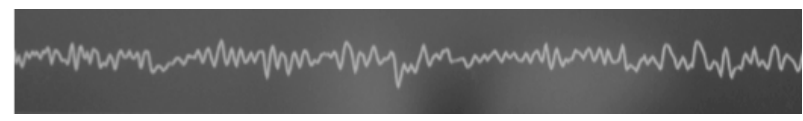

(c)

Figure 1. Bispectral index electroencephalographic raw. (a) Baseline value recorded before carotid cross clamping; (b) Carotid cross clamping and shunt insertion; (c) After mobilization of the shunt.

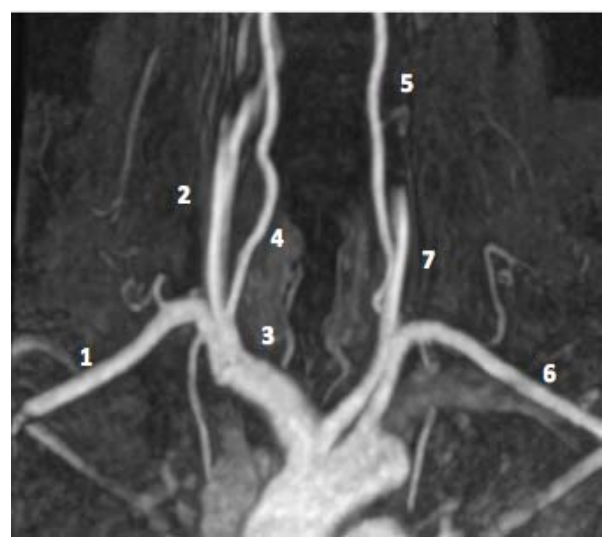

(a)

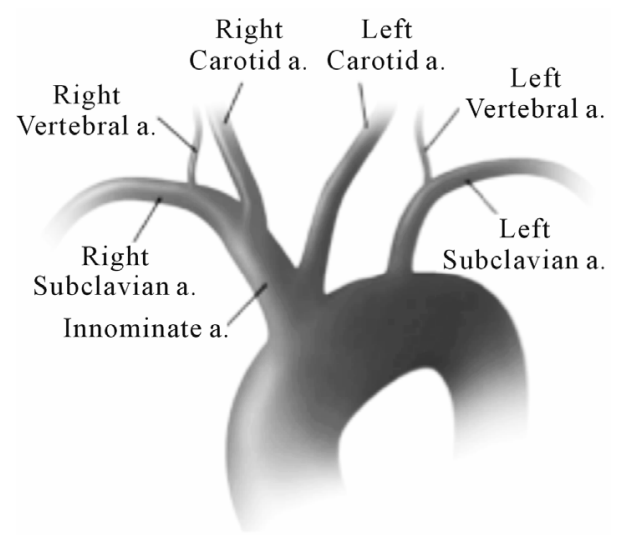

(b)

Figure 2. (a) Magnetic resonance angiography. With 1. Right subclavian artery; 2. Right Carotid artery; 3. Brachiocephalic trunk; 4. Right Vertebral artery; 5 . Left Vertebral artery; 6. Left Subclavian artery; 7. Left Carotid artery. (b) Aortic arch imaging. Reprinted after authors acceptance from Layton KF et al. AJNR 2006; 27: 1541-1542.

period and anesthetic management must be adapted according to this diagnosis.

In the current practice of CEA, anesthesiologists and surgeons usually choose the anesthesia technique they are most comfortable with. Loco-regional anesthesia offers the advantage of direct neurological monitoring of the conscious patient [5]. During general anesthesia, the decision whether or not to insert a temporary carotid shunt depends on the adequacy of the monitoring used. There are a number of techniques and monitors available to detect cerebral ischemia but, until now, none has been shown to improve postoperative outcomes [6]. Indeed, Transcranial Doppler ultrasonography is a sensitive and specific technique that can evaluate flow velocity changes. However, during the perioperative period, intracranial arteries are not easily explored [7]. Somatosensory evoked potentials are good predictor of intraoperative cerebral ischemia but interpretation requires good expertise [8]. Cerebral oxymetry appears to be a promising technique 
but there is no large study showing a very good performance between the percentage of the drop of cerebral oxymetry and cerebral ischemia [9]. Finally, multichannel EEG remains probably the most efficient technique to detect peroperative cerebral ischemia but is still limited by the user expertise, the anesthesia depth, the body temperature, the hemodynamic stability, and last but not least, the position of the electrodes which may not cover all the cerebral territories at risk for ischemia [10].

In this case of left CEA, the continuous multichannel bilateral EEG analysis associated with the interpretation of the modification of the right radial artery waveform allows the early detection of the too deep shunt insertion. The slow drop of the left BIS value (from 45 to 35 ) during this left cerebral ischemic episode could not be considered as clinically relevant.

\section{Conclusion}

In conclusion, this case reports the need of specific management in case of carotid anatomical variation. CEA under general anesthesia requires a careful interpretation of adequate neuromonitoring to avoid poor postoperative outcome.

\section{REFERENCES}

[1] North American Symptomatic Carotid Endarterectomy Trial Collaborators, "Beneficial Effect of Carotid Endarterectomy in Symptomatic Patients with High-Grade Carotid Stenosis," The New England Journal of Medicine, Vol. 325, 1991, pp. 445-453. doi:10.1056/NEJM199108153250701

[2] R. Bond, K. Rerkasem and P. M. Rothwell, "Systematic Review of the Risks of Carotid Endarterectomy in Relation to the Clinical Indication for and Timing of Surgery," Stroke, Vol. 34, 2003, pp. 2290-2301. doi:10.1161/01.STR.0000087785.01407.CC
[3] K. F. Layton, D. F. Kallmes, H. J. Cloft, E. P. Lindell and V. S. Cox, "Bovine Aortic Arch Variant in Humans: Clarification of a Common Misnomer," American Journal of Neuroradiology, Vol. 27, 2006, pp. 1541-1542.

[4] G. C. Jakanani and W. Adair, "Frequency of Variations in Aortic Arch Anatomy Depicted on Multidetector CT," Clinical Radiology, Vol. 65, No. 6, 2010, pp. 481-487. doi:10.1016/j.crad.2010.02.003

[5] N. Attigah, J. Kutter, S. Demirel, M. Hakimi, U. Hinz, J. Motsch and D. Bockler, “Assessment of Patients' Satisfaction in Carotid Surgery under Local Anaesthesia by Psychometrical Testing-A Prospective Cohort Study," European Journal of Vascular \& Endovascular Surgery, Vol. 41, No. 1, 2011, pp. 76-82. doi:10.1016/j.ejvs.2010.08.020

[6] K. Rerkasem and P. M. Rothwell, "Routine or Selective Carotid Artery Shunting for Carotid Endarterectomy (and Different Methods of Monitoring in Selective Shunting)," Cochrane Database of Systematic Reviews, Vol. 4, 2009.

[7] I. C. van der Schaaf, J. Horn, F. L. Moll and R. G. Ackerstaff, "Transcranial Doppler Monitoring after Carotid Endarterectomy," Annals of Vascular Surgery, Vol. 19, No. 1, 2005, pp. 19-24. doi:10.1007/s10016-004-0146-y

[8] P. H. Manninen, T. K. Tan and R. M. Sarjeant, "Somatosensory Evoked Potential Monitoring during Carotid Endarterectomy in Patients with a Stroke,” Anesthesia \& Analgesia, Vol. 91, No. 1, 2001, pp. 39-44. doi:10.1097/00000539-200107000-00010

[9] T. Mille, M. E. Tachimiri, C. Klersy, G. Ticozzelli, G. Bellinzona, I. Blangetti, S. Pirrelli, M. Lovotti and A. Odero, "Near Infrared Spectroscopy Monitoring during Carotid Endarterectomy: Which Threshold Value Is Critical?” European Journal of Vascular \& Endovascular Surgery, Vol. 27, No. 6, 2004, pp. 646-650. doi:10.1016/j.ejvs.2004.02.012

[10] V. Bonhomme, Q. Desiron, T. Lemineur, J. F. Brichant, P. Y. Dewandre and P. Hans, "Bispectral Index Profile during Carotid Cross Clamping,” Journal of Neurosurgical Anesthesiology, Vol. 19, No. 1, 2007, pp. 49-55. doi:10.1097/01.ana.0000211031.49420.c8 